Research Article

\title{
Treatment of severe falciparum malaria: quinine versus artesunate
}

\author{
Dipesh Patel, Vishal Sadatia*, Mehul Patel
}

Department of Medicine, C.U. Shah Medical College and Hospital, Surendranagar 363001, India

Received: 9 November 2012

Accepted: 11 December 2012

*Correspondence to:

Dr. Vishal Sadatia,

Email: drvvsadatia@gmail.com

\begin{abstract}
Background: Malaria is the most important disease of human being. More than $40 \%$ of the world's population is considered to be at risk of exposure of this disease. Malaria infection has been increasing over recent years due to combination of factors including increasing resistance of malarial parasite. Most of the strains of $\mathrm{P}$. falciparum are now resistance to conventional drugs like chloroquine in many areas. The objective of this study was to compare the efficacy and safety of quinine and artesunate in treatment of P. falciparum malaria.
\end{abstract}

Methods: This is hospital based prospective study, conducted amongst 35 randomly selected patients of severe P. falciparum malaria. Patients with any contraindications of either drug were excluded to avoid bias. Standard statistical tests were applied for qualitative as well as quantitative data.

Results: As per the study end point results of difference of mortality in patients receiving either drug was not significant $(p>0.75)$, but difference in clinical parameters like fever clearance time $(p<0.01)$, parasite clearance time $(p<0.001)$ and coma resolution time $(p<0.001)$ were significant among patients receiving artesunate. There were no any significant differences in adverse effects of both the drugs. Mortality was same in both arms taking either drug.

Conclusions: Artesunate is as good as quinine in mortality aspect but artesunate is superior in fever clearance time (FCT) \& parasite clearance time (PCT). Coma resolution time is faster with quinine as compared to artesunate. There are no significant adverse effects of either drug. So artesunate is equivalent or superior for treatment for severe falciparum malaria.

Keywords: Severe falciparum malaria, Quinine, Artesunate

\section{INTRODUCTION}

A protozoan belonging to the genus Plasmodium causes malaria. Four different species cause human infection worldwide. $^{1,2}$ They are 1. Plasmodium Vivax, 2. Plasmodium falciparum, $3 . \quad$ Plasmodium malariae, 4. Plasmodium ovale. Among these four species, P. falciparum causes the most severe forms of malaria and is considered to be the lethal strain. It causes 'Malignant Tertian Malaria' and the severity of this is well known right from the days of Alexander the Great, who was stopped in his tracks by an epidemic during his victorious campaign. Mixed infection due to more than one malarial species occurs in 5 to 7 percent of infections.
Malaria infection has been increasing over recent years due to a combination of factors including increasing resistance of malarial parasites, to chemotherapy, increasing resistance of the Anopheles mosquito vector to insecticides ${ }^{3}$, ecological and climate changes as well as due to increased international travel to malaria endemic areas.

In April 1953 the Govt. of Indian launched the National Malaria Control Programme (NMCP). The NMCP attained quick and commendable results by 1958 . Encouraged by these results, the Govt. of India launched an ambitious programme to eradicate the disease National Malaria Eradication Programme (NMEP). The NMEP continued to attain good results but receiving setbacks by 1965 in the form of increasing frequency of outbreaks 
and increasing annual incidence of malaria cases. Numerous factors were considered to have resulted in this failure like emergence of drug resistant and insecticide resistant strains, migration of people so in the late nineties this was renamed the National Anti-Malarial Programme (NAMP). ${ }^{4}$

Most of the strains of P. falciparum are now resistant to conventional drugs like chloroquine in many endemic areas including India. As a result of enthusiastic research we now have drugs like Qinghaosu derivatives, Atovaquone, Bulaquine, Proguanil, Mefloquine, Pyronaridine, and Halofantrine. ${ }^{5-10}$ Of concern also, is to identify if the newer antimalarials are better than the time tested ones in terms of saving lives and promoting a hasty recovery. Further clinical trials are warranted to test the newer antimalarials and this is a humble effort in that direction.

\section{METHODS}

All patients included in the study were above 18 years of age and were admitted in the medical wards of C.U. Shah Hospital between October 2011 to April 2012. The patients were randomly allocated to one of the two groups, one which received quinine and the other which received artesunate as the antimalarial drug.

Thirty five patients were included in this study according to following inclusion and exclusion criteria.

\section{Inclusion criteria:}

1. Adult patients admitted to the medical wards of hospital with the history of high grade fever with chills and rigor.

2. Presence of asexual form of P. falciparum in the peripheral smear.

3. Patients satisfying at least following criteria for establishing a diagnosis sever P. falciparum (adopted from the WHO definition of sever malaria):

a. Cerebral malaria: Unarousable coma not attributable to any other cause in a patient with falciparum malaria, lasting for $>40$ minutes, if following seizure.

b. Sever normochromic normocytic anemia: $\mathrm{Hb}$ level $<5 \mathrm{gm} / \mathrm{dl}$ or a hematocrit $<15 \%$ in the presence of a parasitemia level above $10000 / \mu \mathrm{L}$

c. Renal failure: urine output $<400 \mathrm{ml}$ (or 12 $\mathrm{ml} / \mathrm{kg}$ ) in $24 \mathrm{hrs}$, failing to improve after rehydration or a creatine value $>3 \mathrm{mg} / \mathrm{dl}$.

d. Acute Respiratory Distress Syndrome (ARDS).

e. Hypoglycemia: whole blood glucose level < $40 \mathrm{mg} / \mathrm{dl}$.

f. Spontaneous bleeding from the gum, nose, GIT or substantial laboratory evidence of Disseminated Intravascular Coagulation (DIC). g. Repeated generalized convulsion> 2 in 24 hrs, despite adequate cooling if fever.

h. Acidosis: arterial $\mathrm{PH}<7.25$ or bicarbonate, $15 \mathrm{mmol} / \mathrm{L}$.

\section{Exclusion criteria:}

1. Hypotension at the time of presentation.

2. Electrocardiograph showing a corrected QT (QTc) interval of 0.45 seconds.

3. Glucose 6 phosphate dehydrogenase deficiency (G6PD).

4. Multiple species infestation.

5. Patients who have received anti-malarial medication prior to admission for the present illness.

6. Patient admitted in a stage of the illness with parasitemia level of $>106 / \mu 1$ and dying within 24 hrs. of admission.

\section{Primary end points:}

- Death was the primary end point in the study.

\section{Secondary end points:}

- Fever clearance time (FCT)

- Parasite clearance time (PCT)

- Coma resolution time (CRT)

- Adverse effects of drugs.

Dosage in the quinine arm: Patients in the group received a loading dose of $20 \mathrm{mg} / \mathrm{kg}$ infused over $6 \mathrm{hrs}$ followed by 6 hour infusions of $10 \mathrm{mg} / \mathrm{kg}$ every 8 hourly with a maximum dose of $1800 \mathrm{mg}$ in the first $24 \mathrm{hrs}$. In patients who continued to remain in the severe state of the disease for more than $48 \mathrm{hrs}$, the dose of Quinine was reduced by fifty percent.

Dosage in the artesunate ${ }^{11}$ arm: The patients in this group were given Artesunate in the standard dose of 2.4 $\mathrm{mg} / \mathrm{kg}$ on day 1 followed by $1.2 \mathrm{mg} / \mathrm{kg}$ OD for six more days completing a total dose of $9.6 \mathrm{mg} / \mathrm{kg}$.

A thorough clinical and laboratory examination of the patients was conducted. The temperature, pulse, respiration and blood pressure were recorded at the time of admission, every 4 hourly thereafter and as and when required. The systemic examination was done daily and the Central nervous system examination was repeated at the time of regaining consciousness in case of cerebral malaria patients. The fundus examination was also done daily in all patients.

The end points of the study, stated previously, were analyzed systematically and efficacy of the drugs was compared using statistical methods which are described in detail later. 


\section{Working definition of end points ${ }^{12}$}

- Parasite Clearances Time(PCT) : Time (in hours) since the initiation of therapy to the first negative blood smear

- Parasite Clearance Time ${ }^{50}\left(\mathrm{PCT}^{50}\right)$ : Time (in hours) since the initiation of therapy to $50 \%$ reduction in the parasitemia level.

- Parasite Clearance Time ${ }^{75}\left(\mathrm{PCT}^{75}\right)$ : Time (in hours) since the initiation of therapy to $75 \%$ reduction in the parasitemia level.

- Fever Clearance Time A (FCT A) : Time ( in hours) since the initiation of therapy to the first time the patient's temperature goes below $99 \mathrm{~F}$.

- Fever Clearance Time B (FCT B) : Time ( in hours) since the initiation of therapy to the time the patient's temperature goes below $99 \mathrm{~F}$.

- Coma Resolution Time (CRT): Time ( in hours) since the initiation of therapy to the time the patient was fully conscious with a GCS of 15

- Probable Adverse Reaction: A probable adverse reaction to the drug is one which follows a reasonable temporal sequence from administration of the medicine; that follows a known or expected response to the drug; and that could not be reasonably explained by the known characteristics of the disease state.

\section{RESULTS}

Thirty five patients following scrupulous scrutiny of the inclusion and exclusion criteria were selected. On randomization, eighteen patients were included in the Quinine arm while seventeen were included in the Artesunate arm. Thirty three patients completed the study as per study protocol. Two patients succumbed to the illness, one each in the Artesunate and Quinine arms. There was also no significant side effect to any of the two drugs and there was no crossover from one arm to the other during the study. Two patients in each of the two arms developed ARDS and were shifted to the ICU.
The breakup of the different manifestations of severe falciparum malaria in both groups is depicted along with their percentages in figure 1. In the Quinine arm coma was present in 11 patients, while 7 patients had acute renal failure. Thrombocytopenia was seen in 6 patients and severe anemia in 1 patient. Significant jaundice was seen in 10 patients, while only 2 in the arm had ARDS. In the Artesunate arm there were 11 patients with coma, 9 with renal failure, 5 with thrombocytopenia and 1 with severe anemia. There were 10 patients with severe Jaundice and 2 with ARDS.

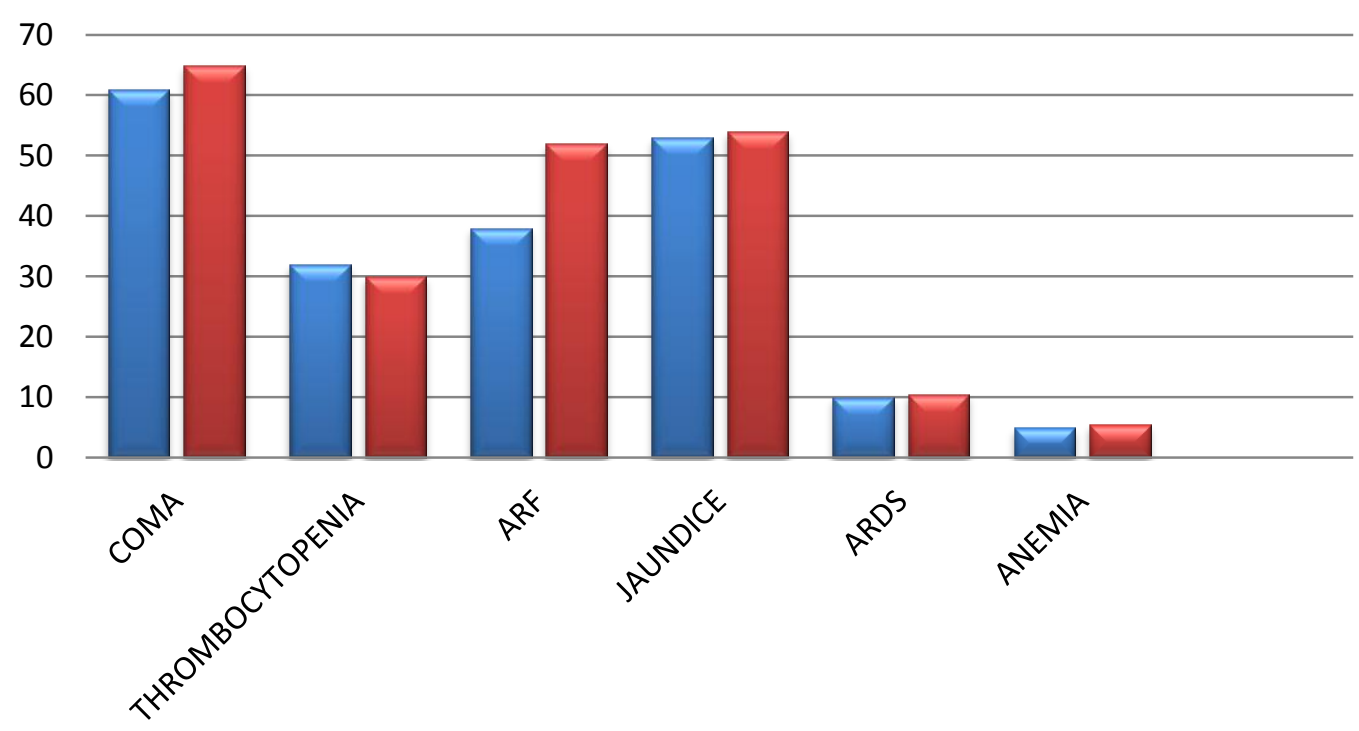

$\square$ QUININE GROUP $\square$ ARTESUNATE GROUP

Figure 1: Disease manifestations in two groups. 


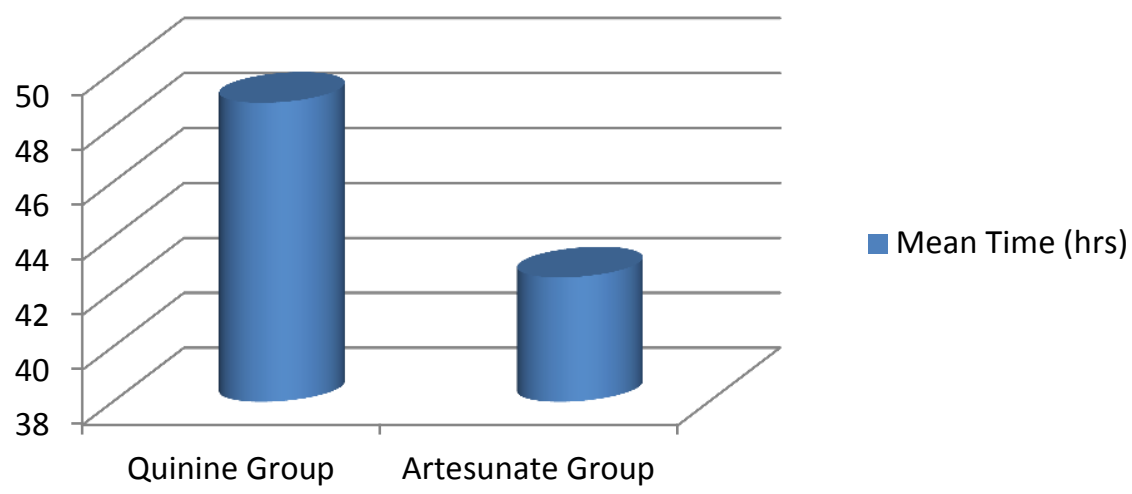

Figure 2: Mean FCT in the two groups.

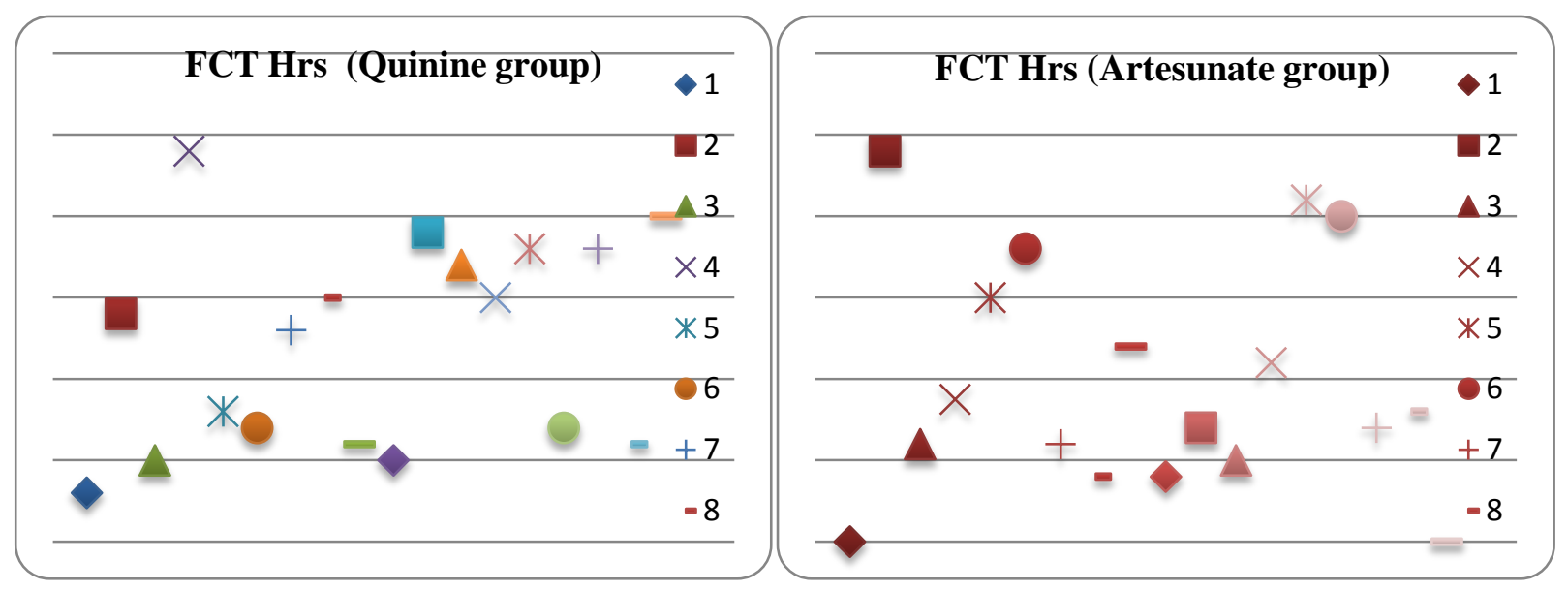

Figure 3: The scatter diagram shows the clustering points in the higher range.

Table 1: Parasite burden on presentation.

\begin{tabular}{|c|c|c|c|c|}
\hline Factor & $\begin{array}{l}\text { Quinine } \\
\text { Arm }\end{array}$ & $\begin{array}{l}\text { Artesunate } \\
\text { Arm }\end{array}$ & t value & $P$ value \\
\hline Mean & $37924 / \mu \mathrm{L}$ & $36665 / \mu \mathrm{L}$ & \multirow{3}{*}{0.703662} & \multirow{3}{*}{$>0.40$} \\
\hline Range & $640-74000 / \mu \mathrm{L}$ & $1200-70000 / \mu \mathrm{L}$ & & \\
\hline SD & 19852 & 20655 & & \\
\hline
\end{tabular}

Table 2: Fever clearance time.

\begin{tabular}{|lllll|} 
Factor & Quinine Arm & Artesunate Arm & t value & p value \\
\hline Mean & $48.89 \mathrm{hrs}$ & $42.53 \mathrm{hrs}$ & & \\
\cline { 1 - 3 } Range & $12-96 \mathrm{hrs}$ & $16-96 \mathrm{hrs}$ & & \\
\hline SD & 25.54 & 26.32 & & \\
\hline
\end{tabular}


Table 3: Parasite clearance time.

\begin{tabular}{|c|c|c|c|c|c|}
\hline Factor & & Quinine Arm & Artesunate Arm & t value & $p$ value \\
\hline \multirow{3}{*}{$\mathrm{PCT}^{50}$} & Mean & $17.33 \mathrm{hrs}$ & 13.177 & \multirow{3}{*}{5.140453} & \multirow{3}{*}{$<0.001$} \\
\hline & Range & $8-32$ hrs & $8-32$ hrs & & \\
\hline & SD & 7.8815 & 6.894 & & \\
\hline \multirow{3}{*}{$\mathbf{P C T}^{75}$} & Mean & $32.44 \mathrm{hrs}$ & $28.43 \mathrm{hrs}$ & \multirow{3}{*}{1.974683} & \multirow{3}{*}{$>0.05$} \\
\hline & Range & $12-48 \mathrm{hrs}$ & $12-48 \mathrm{hrs}$ & & \\
\hline & SD & 14.051 & 11.535 & & \\
\hline \multirow{3}{*}{$\mathrm{PCT}^{100}$} & Mean & $51.11 \mathrm{hrs}$ & $43.765 \mathrm{hrs}$ & \multirow{3}{*}{2.048062} & \multirow{3}{*}{$<0.05$} \\
\hline & Range & $16-96 \mathrm{hrs}$ & $16-84 \mathrm{hrs}$ & & \\
\hline & SD & 24.94 & 19.21 & & \\
\hline
\end{tabular}

Table 4: Coma resolution time.

\begin{tabular}{|c|c|c|c|c|}
\hline Factor & Quinine Arm & Artesunate Arm & t value & $p$ value \\
\hline Mean & $19.091 \mathrm{hrs}$ & $25.818 \mathrm{hrs}$ & \multirow{3}{*}{3.85204} & \multirow{3}{*}{$<0.001$} \\
\hline Range & $06-60 \mathrm{hrs}$ & 08-64 hrs & & \\
\hline SD & 16.232 & 17.285 & & \\
\hline
\end{tabular}

\section{Parasite burden on presentation}

The parasite load in both groups was comparable before initiation of therapy. This was estimated, like other factor, because it has a bearing on the outcome of drug therapy. The mean parasite loads in the two groups are shown in table 1 below the difference noted was not statistically significant $(p>0.40)$.

\section{Analysis of end points}

The primary end point of the study was death while the secondary end points were the fever clearance time, parasite clearance time and the coma resolution time. Each parameter was analyzed separately.

Fever Clearance Time (FCT): The fever clearance time (FCT) has already been defined. Two form of fever clearance time have been described in literature available FCTA and FCTB.

Table 2 shows a low FCT in the patients treated with Artesunate. The ranges as well as SD of the groups were similar. On performing the $\mathrm{t}$ test the difference in the means were found to be significant $(p<0.001)$.

Parasite Clearance Time (PCT): The ability of the two drugs to cause parasitemia clearance was estimated and compared. The time taken to clear the parasites to $50 \%$ and $75 \%$ of the initial burden was also estimated to assess the efficacy of the drugs throughout the course of therapy.

Table 3 shows the mean PCT of the patients treated with Quinine to be $51.11 \mathrm{hrs}$ while that of the Artesunate group was only $43.765 \mathrm{hrs}$ a similar but more significant difference was seen in the PCT50 of the two groups indicting a more rapid clearance of the parasites patients treated with Artesunate with an efficacy to clear the parasitemia rapidly even in the initial heavy burden state. The PCT75 of the two groups were $32.44 \mathrm{hrs}$ and 28.94 hrs respectively in the Quinine and the Artesunate group. Though the values shows better result with Artesunate this failed to prove true in the test of significance.

To get a visual impression of the difference in the efficacy of parasitemia clearance in the two groups, a smooth line graph plotted as percentage parasitemia remaining against time since starting drug therapy.

Figure 4 shows the population parasite clearance curve of the two groups. The population parasite clearance curve refers to the average of all the individual parasite clearance curves. It is clearly seen that the patients in the Quinine group take longer time for complete parasite clearance. If the areas between the two graphs are noted there is wide separation initially at the $50 \%$ clearance mark, which reduces as it reaches the $75 \%$ clearance mark, only to increase again at the $100 \%$ clearance mark. The area between the two curves symbolizes the additional clearance of parasitemia with Artesunate.

Coma Resolution Time (CRT): The coma resolution time (CRT) or the time to regain consciousness, with a GCS of 15, was observed in the two groups and analyzed. A significantly lower CRT was observed in the patients who had received Quinine. The observations made in this regard are depicted in table 4. 


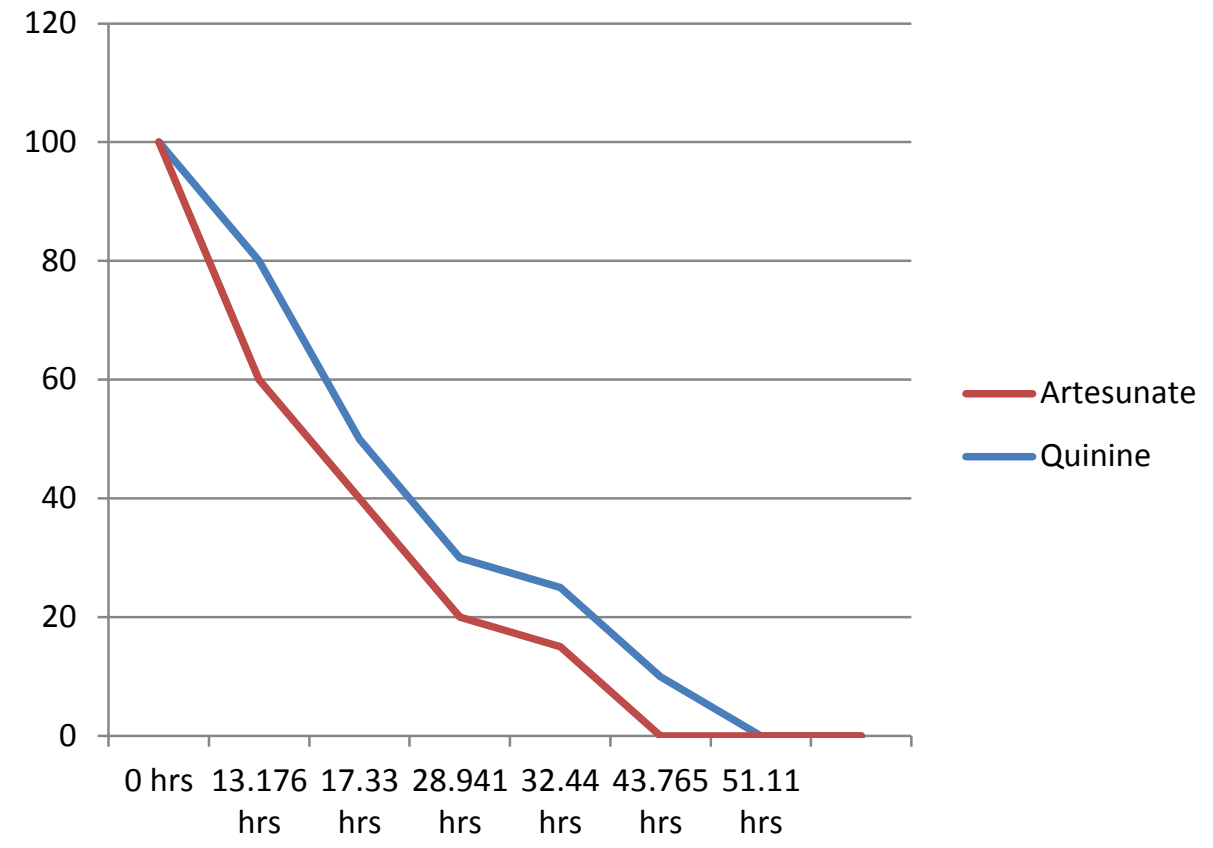

Figure 4: Parasitemia clearance in the two groups with relation to initiation of therapy.

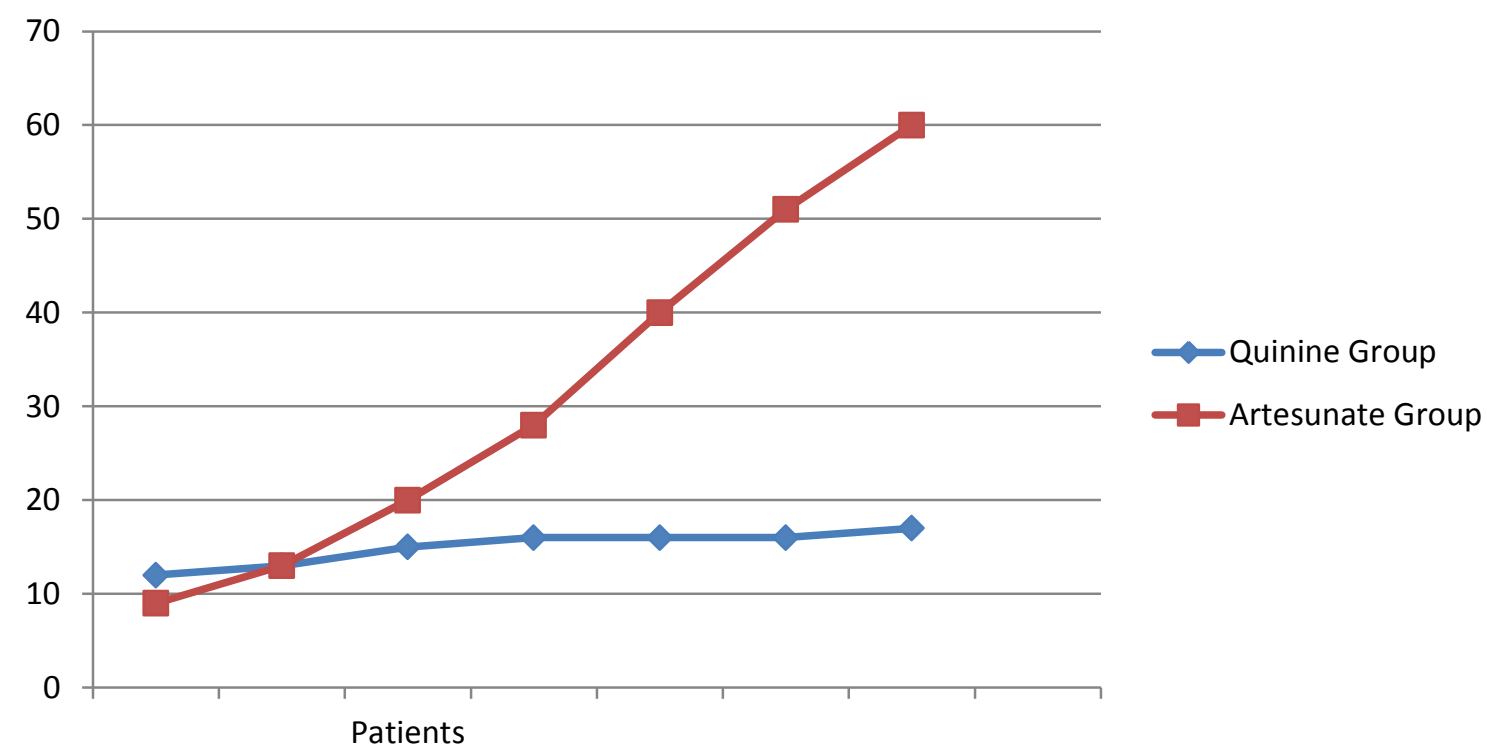

Figure 5: Coma resolution time.

It is evident that the patients in the Quinine arm had a lower mean CRT (19.1 hrs vs. 25.8 hrs) which was statistically ( $p$ value $<0.001$ ). To depict this CRT of individual patients was plotted and a trend line used for each arm in figure 5.

Mortality: One patient in each arm expired during the study. The mortality rate in the Quinine arm was $5.55 \%$ and that Artesunate arm was $5.882 \%$. On chi-square test, the $\mathrm{x} 2$ value was 0.06102 and the $\mathrm{p}$ value was $>0.75$.
Hence there was no statistically significant difference in mortality between two groups.

The patient who died in the Quinine group had severe malaria with ARDS with severe metabolic acidosis with jaundice; he however had achieved more than 50\% clearance of parasitemia by $16 \mathrm{hrs}$ and cleared it completely by $32 \mathrm{hrs}$. A second smear was not possible, after the first negative smear, as the patient expired before $40 \mathrm{hrs}$ form initiation of therapy. 
In the Artesunate arm the patient who died had cerebral malaria with jaundice with ARF with severe metabolic acidosis. Complete parasite clearance was achieved by 72 hrs but the renal and liver function tests fails to return to normal. The patients gained consciousness by $64 \mathrm{hrs}$ and fever clearance was achieved by 96 hrs.

\section{Adverse effects}

There were no significant adverse effects noted during the study to any of the two drugs. Both groups were closely monitored for hypoglycemia, hypertension, neurological effects, ECG abnormalities, systemic side effects etc. Although none of them caused a significant prolongation of the QTc, enough to warrant withdrawal of the drug.

\section{DISCUSSION}

The result of this study shows that there was no significant difference between the two groups in mortality $(p>0.75)$. The fever clearance time (FCT) was significantly lower in the group of patients treated with Artesunate $(p<0.01)$. The $50 \%$ parasite clearance rate (PCT) \& complete parasite clearance time (PCT) was low $(p<0.001)$ in the group treated with Artesunate. The coma resolution time (CRT) was significantly lower in the Quinine arm compared to the Artesunate arm ( $p$ $<0.001)$. There were no significant adverse effects with both drugs.

The better response to Artesunate reflects the better absorption of the drug. ${ }^{11}$ Artesunate also has ability to prevent merogony by the later stages of the parasites ${ }^{7}$, which prevent the continuing increase in parasitemia in spite of therapy with other drugs. Another explanation is the clearance of RBCs infected with the ring forms. This could be due to oxidative membrane damage or splenic clearance.

A significantly lower CRT was observed in the patients who had received Quinine compared to Artesunate. The exact reason for this is controversial. This could be due to the neurotoxicity of the Artemisinin compounds due to which the patients take a longer time to recover. It is yet to be proved whether Quinine has some sort of anticytokine effect or other cause which might put it in favour in cerebral malaria patients. Chloroquine has already been proved have an anti TNF $\alpha$ action in the body. Another possible explanation is that the Artemisinin compounds are actually saving the severe patients, who would have otherwise died in the Quinine arm, at the cost of prolonged CRT.
In spite of the faster parasite clearance observed with Artesunate, there was no significant mortality advantage associated with its use. One way of looking at this paradox is Artesunate may be actually preferentially clearing the younger parasites seen in the peripheral smear compared to the sequestrated parasites, which actually cause organ damage.

To conclude Artesunate was as good as Quinine in the treatment of severe falciparum malaria. As far as cerebral malaria is concerned, Quinine is preferable as it is having better recovery from coma.

\section{Funding: No funding sources \\ Competing interests: None declared}

Ethical approval: The study was approved by the Institutional Ethics Committee

\section{REFERENCES}

1. Epidemiology of communicable diseases, in Park's textbook of preventive and social medicine. K. Park (Ed) 18th edition. Jabalpur, Banarasidas Bhanot Publish. 2008:198-200.

2. Roberts L. Mosquitoes and disease. Science 2002;298:82-3

3. Hemingway J, Field L, Vontas J. An overview of insecticide resistance. Science 2002;298:96-7.

4. National anti-malaria programme: Strategy and Policies. In malaria and its control in India. Country Scenario: March 1999. National Anti-Malarial Programme (NAMP), DGHS, Govt. of India, 1999:1-11.

5. Sachs J, Malaney P. The economic and social burden of malaria. Nature 2002;415:680-5.

6. Sachs JD. A new global effort to control malaria. Science 2002;298:122-4.

7. Kyes S, Horrocks P, Newbold C. Antigenic variation at the infected red cell surface in malaria. Annu Rev Microbiol 2001;55:673-707.

8. Greenwood B, Mutabingwa T. Malaria in 2002. Nature 2002;415:670-2.

9. White NJ. The treatment of malaria. N Engl J Med 1996;335:800-6.

10. Pinswadi K, Chaereonkwan P. Sluggish response of a case of falciparum malaria to intensive quinine therapy. J Med Assoc Thai 1965;48:386.

11. Barradell LB, Fitton A. Artesunate. Drugs 1995;50:714-41.

12. World Health Organization. Severe and complicated malaria. World Health Organization, Division of Control of Tropical Diseases. Trans R Soc Trop Med Hyg 1990;84 Suppl 2:1-65.

doi: 10.5455/2319-2003.ijbcp20130106

Cite this article as: Patel D, Sadatia V, Patel M.

Treatment of severe falciparum malaria: quinine versus artesunate. Int J Basic Clin Pharmacol

2013;2:30-6. 